\title{
What masks utrocular discrimination
}

\author{
WILLIAM MARTENS, RANDOLPH BLAKE, and MICHAEL SLOANE \\ Cresap Neuroscience Laboratory, Northwestern University, Evanston, Illinois 60201 \\ and \\ ROBERT H. CORMACK \\ New Mexico Institute of Mining and Technology, Socorro, New Mexico 87801
}

\begin{abstract}
Utrocular discrimination, the ability to judge which eye has received monocular stimulation, was studied under conditions designed to reduce the salience of the cue mediating this discrimination. In one series of experiments, these conditions involved reducing the likelihood of motor command signals, overwhelming the motor command centers, and triggering motor command signals for both eyes. Those results indicate that utrocular discrimination is not mediated by ocular movements or command signals. In a second series of experiments, a visual masking paradigm was used. A monocular test grating appeared superimposed upon a background of binocular masking noise, which was presented either simultaneously with the target or at some interval preceding or following the target (asynchronous masking). When presented simultaneously, the binocular masking noise interfered with utrocular performance, but only when the noise contained spatial frequencies near the test frequency. Masking functions obtained under conditions of asynchronous masking indicated that the time course of the forward and backward masking changed with spatial frequency. Finally, a sustained masking pattern that was ineffective in reducing utrocular performance could be made effective by pulsing the mask simultaneously with the target. Taken together, these results implicate a transient response to the target onset as a critical factor in generating the cue for successful utrocular discrimination. This conclusion may account for the fact that utrocular discrimination falls off for normal observers as the spatial frequency of the target grating increases above 4 cycles/deg.
\end{abstract}

Recently we have become interested in the problem of utrocular discrimination (UD), the ability of an observer to judge which eye has received monocular stimulation. This interest stems from the desire to develop a psychophysical procedure for studying ocular dominance systems in human vision, that is, groups of neurons differing in their responsiveness to stimulation of one eye with respect to the other (e.g., Hubel \& Wiesel, 1968). It is this physiological property that provides a plausible neural basis for the psychophysical phenomenon of UD.

Previous work in this laboratory has identified a set of conditions under which most observers are able to perform UD successfully (Blake \& Cormack, 1979). In particular, when the monocular target consists of a sinusoidal grating, most (but not all) observers have no trouble judging which eye receives the pattern, provided the spatial frequency of the grating is less than 4 cycles/deg; for values of spatial frequency greater than this, UD performance deteriorates rapidly.

Having identified stimulus conditions sufficient for UD, we are now in the position to investigate in

This research was supported by Grants BNS7817948 from NSF and EY01596 from NIH. R.B. holds a Career Development Award from NIH (EY00106). We thank Geoffrey Iverson for his many helpful comments on an earlier version of this paper. more detail the nature of the neural process responsible for this ability. Toward that end, the experiments described in this paper sought to determine under what conditions the normally potent cue for UD could be weakened or abolished, as evidenced by a drop in UD performance. In one series of experiments (Section 1), we explored the possibility that UD is mediated by some form of oculomotor cue accompanying monocular stimulation. Having rejected this hypothesis, we next performed a series of masking experiments (Section 2) to determine the spatial and temporal properties of the mechanism responsible for UD.

This paper is organized in the following way. First, methodological details common to all the experiments are described. Next comes the section dealing with the question of oculomotor involvement in UD, and this is followed by the section on visual masking of UD. Each of these sections is introduced by the rationale behind those experiments. The paper concludes with a working model of the neural events underlying UD.

\section{GENERAL METHODS}

\section{Apparatus and Displays}

The details of the apparatus are fully described elsewhere (Blake \& Cormack, 1979). In summary, two matched CRT displays 
( $7 \times 5$ deg visual angle) were presented separately to the eyes via a mirror stereoscope. Rotation of a pair of front-surface mirrors allowed the observer to bring the two CRT displays into proper binocular alignment. The -observer viewed these displays in an otherwise darkened test chamber, with the head firmly positioned on a dental impression board.

Conventional electronic techniques were used to generate vertical sinusoidal grating patterns and one-dimensional noise. Using electronic shaping circuitry, grating contrast could be spatially vignetted such that modulation was zero at the borders. The display could be briefly presented with any desired temporal onset and offset by passing the electronic signal through a programmable rise-fall gate before applying the signal to the $Z$ axis of the CRT. The gates could be triggered by a buttor that was under the observer's control. Introduction of a grating or noise had no influence on the average luminance, which was $7 \mathrm{~cd} \mathrm{~m}^{-2}$. Contrast could be varied in 1-dB (.05-log-unit) steps using precision attenuators.

\section{Procedure}

In all experiments, a two-alternative, forced-choice procedure was used to determine percent-correct discrimination. Each trial was initiated when the observer pressed a button that triggered the brief presentation of a pattern to one display or the other. The observer used a two-position manual switch to indicate which eye was stimulated, guessing when necessary. Following each trial, the observer was provided with feedback, in the form of a tone, as to the correctness of the response. The eye receiving stimulation was varied randomly over trials, which were typically administered in blocks of 100 for a given stimulus condition. Data were analyzed for the two eyes separately, but, since performance was always virtually identical for the two eyes, results have been pooled for purposes of description.

Great care was taken to insure proper binocular alignment of the two displays and to eliminate potential extraneous cues (e.g., differences in apparent contrast between the left-eye and right-eye patterns).

\section{Observers}

For the purposes of the experiments reported here, we needed observers who could reliably perform UD, since our intent was to investigate the nature of the cue mediating successful performance. For that reason, two of the authors (R.B. and W.M.) served as observers. Besides having excellent visual acuity $(20 / 20$ or better for both eyes) and good stereopsis, these individuals are proficient and well-practiced at UD.

\section{OCULOMOTOR INVOLVEMENT IN UTROCULAR DISCRIMINATION}

An account occurring frequently in the literature on UD has been that the effective cue seems to be a strain or feeling in the stimulated eye (Blake \& Cormack, 1979; Enoch, Goldmann, \& Sunga, 1969; Smith, 1945). Clearly, these sorts of descriptions suggest a kinesthetic cue, which immediately raises the possibility that ocular reflexes provide the cue for UD. Not surprisingly, several investigators have tested UD under conditions of cycloplegia, to paralyze accommodation and pupillary reflexes and thereby eliminate these as potential cues (Blake \& Cormack, 1979; Smith, 1945). This maneuver, however, has proved ineffective in disturbing successful UD or in diluting the strength of the mediating cue. There is some evidence (Smith, 1945) that forced convergence can impair but not abolish UD, which could implicate extrinsic musculature as a source for cues. It is conceivable that monocular stimulation triggers a vergence error signal leading to some weak eye movements, although there is certainly no visual concomitant to suggest such an event. Another possibility is that information for UD comes, not from kinesthetic feedback, but instead from command centers responsible for initiation of eye movements or ocular reflexes. Now, obviously, it is rather more difficult to isolate the effects of command signals than it is to study eye movements directly. However, several strategies seemed promising. One approach would be to test UD using stimulus conditions unlikely to initiate motor command signals. Alternatively, one might attempt to overwhelm the system by keeping the command centers very active during the UD trials, thus masking any potential cue for UD. Finally, one might try to insure that both eyes are stimulated equally so that comparable command signals are associated with each eye. We have attempted all three approaches.

\section{Stimuli Less Likely to Initiate \\ Monocular Motor Commands}

In most UD experiments, monocular stimulation is delivered to an eye that is otherwise viewing a homogeneous field. This abrupt introduction of visual input would seem to provide a very strong stimulus for ocular reflexes that, in turn, could furnish information about the eye stimulated. On the other hand, if both eyes were already engaged by stimulation, a decrement in one eye's input might be less likely to produce reflexive oculomotor events and, therefore, be less salient as a cue for UD. In fact, Templeton and Green (1968) reported that observers were indeed unable to judge which member of a pair of fused circular spots of light was briefly extinguished. However, Templeton and Green did not measure UD under more conventional conditions, so we do not know to what extent their failure to find successful UD with decrements supports an oculomotor hypothesis. Our first experiment was designed to provide a more generous test of this hypothesis.

In this first experiment, we employed a variation of Templeton and Green's paradigm, which we refer to as the asynchronous of fset procedure. Observers viewed a pair of fused grating patterns. When a trial was triggered, one of the gratings disappeared, leaving an uncontoured raster in one eye. After a brief interval, referred to as the stimulus offset asynchrony (SOA), the other grating was removed, thus leaving both screens blank. Following a second interval $(500 \mathrm{msec})$, both gratings reappeared simultaneously. The observer's task was to identify from which eye the grating was first removed. This procedure eliminates the onset of a monocular stimulus, a maneuver that we hoped would minimize the initiation of a distinctive motor command.

Observers R.B. and W.M. executed 100 trials at each of 10 SOAs. Spatial frequency was $1 \mathrm{cycle} / \mathrm{deg}$, a value at which both observers can discriminate very 


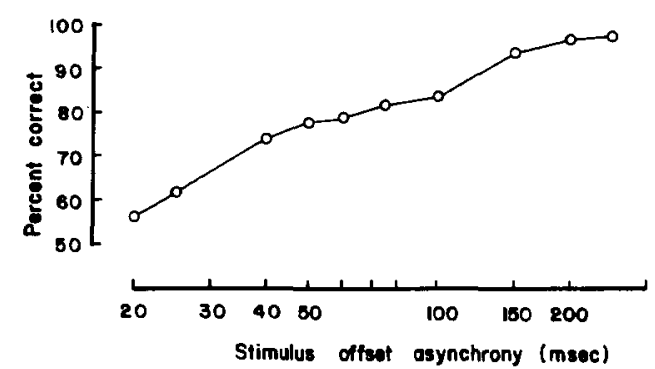

Figure 1. Utrocular discrimination as a function of stimulus offset asynchrony for Observer R.B. Observer W.M. shows the same pattern of results. $\mathbf{N}=100$.

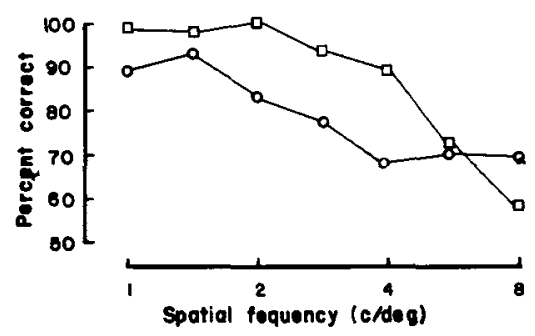

Figure 2. Utrocular discrimination in an asynchronous offset procedure as a function of spatial frequency for Observers R.B. (squares) and W.M. (circles). $\mathrm{N}=100$.

reliably which eye receives the onset of a grating pattern. Contrast was $10 \%$, and the rise-fall gate was set to its minimum value $(1 \mathrm{msec})$. The typical results are shown in Figure 1. At an SOA of $250 \mathrm{msec}$, performance was nearly perfect and remained above chance down to $25 \mathrm{msec}$.

We know that utrocular discrimination for briefly presented grating patterns is strongly dependent on spatial frequency (Blake \& Cormack, 1979). To determine whether the same dependence obtains for asynchronous offset, Observers R.B. and W.M. performed 100 trials at each of seven spatial frequencies. Contrast was $10 \%$, SOA equaled $350 \mathrm{msec}$, and the rise-fall time was $150 \mathrm{msec}$. The results, presented in Figure 2, parallel closely those obtained in experiments using the more conventional monocularly presented grating patterns, in that UD is dependent on spatial frequency.

\section{Overwhelming Oculomotor Command Centers}

In this series of experiments, we returned to the conventional UD paradigm involving brief grating presentation to one eye and tested under conditions designed to maintain activity within various oculomotor systems. By so doing, we hoped to swamp any motoric cue occasioned by the introduction of the test target. In order to stimulate the accommodative system during UD, Observer R.B. viewed the displays through 2.75-D concave lenses. Conditions were 1 cycle/deg, $10 \%$ contrast, 350 msec duration, $150 \mathrm{msec}$ rise-fall time. Despite this extreme forced accommodation, R.B. still achieved $86 \%$ correct for 100 trials, a level of performance comparable to that obtained under conditions of more relaxed accommodation.

Forced convergence also failed to interfere with UD. Conditions were identical to those for forced accommodation except that the lenses were replaced by 5.5-D base-out prisms, thereby requiring $11 \mathrm{D}$ of convergence. At 1 cycle/deg, R.B. achieved $95 \%$ correct for 100 trials. At 8 cycles/deg, performance dropped to $45 \%$ correct, which is the chance level typically measured at this spatial frequency. This failure to perform at 8 cycles/deg is important, for it shows that the success achieved at 1 cycle/deg was not due to differential distortions of the monocular views produced by the different prism orientations for the two eyes.

We attempted to keep the fixation mechanism occupied by requiring observers to track a moving fixation point while attempting UD. In this experiment, when the observer triggered a trial, a small 9-min spot of light began moving horizontally across the display at uniform velocity; the spot was presented to the left eye only. The observer was instructed to maintain fixation of the spot as it moved across the display. The test grating was presented when the dot was near the center of the display.

Observers R.B. and W.M. performed 100 trials at each of three spot velocities $(2.33,6$, and $11.5 \mathrm{deg} / \mathrm{sec})$ and at two spatial frequencies (1 cycle/deg and 8 cycles/deg). Contrast was $10 \%$, exposure duration was $350 \mathrm{msec}$, and rise-fall time was $150 \mathrm{msec}$. The results are shown in Table 1. Performance at 8 cycles/ deg remained around the chance level for both observers for all fixation-point velocities. At 1 cycle/ deg both observers exhibited a decline in performance as tracking velocity increased, but even at $11.5 \mathrm{deg} / \mathrm{sec}$ performance remained significantly above chance. Control trials involving steady fixation established that movement of the dot across the grating was itself insufficient to influence UD; the decline in performance was contingent on tracking

Table 1

Percent Correct U.D. Performances for Three Ocular Tracking Velocities (TV) at Two Spatial Frequencies for Two Observers

\begin{tabular}{|c|c|c|c|c|c|c|}
\hline \multirow[b]{3}{*}{$\mathbf{O}$} & \multicolumn{6}{|c|}{ Spatial Frequency } \\
\hline & \multicolumn{2}{|c|}{$\mathrm{TV}=2.33$} & \multicolumn{2}{|c|}{$\mathrm{TV}=6$} & \multicolumn{2}{|c|}{$\mathrm{TV}=11.5$} \\
\hline & 1 & 8 & 1 & 8 & 1 & 8 \\
\hline $\begin{array}{l}\text { R.B. } \\
\text { W.M. }\end{array}$ & $\begin{array}{l}97 \\
93\end{array}$ & $\begin{array}{l}46 \\
57\end{array}$ & $\begin{array}{l}87 \\
71\end{array}$ & $\begin{array}{l}56 \\
54\end{array}$ & $\begin{array}{l}76 \\
61\end{array}$ & $\begin{array}{l}47 \\
50\end{array}$ \\
\hline
\end{tabular}

Note $-N=100$. Spatial frequencies are given in cycles per degree. Tracking velocities are given in degrees per second. $O=$ observer. 
the dot. Separate analysis of data for each eye showed no differences, hence ruling out any differential effect of the monocularly presented dot.

\section{Equal Stimulation for Both Eyes}

Another approach designed to prevent unilateral motor commands involves simultaneous presentation of discriminable stimuli to both eyes. The observer is required to identify which eye received one of the stimuli. A version of this procedure was employed in our earlier work, in which a high-contrast grating pattern was presented to one eye ard a low-contrast grating was presented to the other eye. Observers who could make UD under the usual conditions of monocular stimulation could also perform successfully at this task, as long as the contrast difference between the two eyes exceeded $8 \mathrm{~dB}$ (Blake \& Cormack, 1979). The use of contrast as the dimension to be discriminated is problematic in the present context, for it could be argued that impulses from the eye receiving the higher contrast grating pattern arrive at relevant neural centers earlier than those from the other eye or that the eye receiving the lower contrast pattern is overwhelmed by the more strongly stimulated eye. For the present purposes, we need a dichoptic stimulus in which the energic or information values are quantitatively similar. Toward this end, we have employed differences in spatial frequency to provide distinctive but quantitatively equivalent stimulation to the two eyes.

Observers judged which of the two eyes received the lower spatial frequency. Both eyes received horizontal sine-wave grating patterns at $10 \%$ contrast; horizontal patterns were used to avoid the potential cue provided by the stereoscopic tilt effect that occurs when vertical gratings of different spatial frequencies are presented to the two eyes. Stimulus duration was $350 \mathrm{msec}$, and rise-fall time was $150 \mathrm{msec}$. One eye, randomized across trials, received a 1-cycle/ deg pattern. For each block of 100 trials, the other eye received $1.09,1.19,1.41,2,2.83,4,5.66$, or 8 cycles/deg.

The results for both observers are presented in Figure 3. When the spatial frequency disparity equaled or exceeded $.15 \mathrm{log}$ units, performance was well above chance. For smaller disparities, UD performance dropped dramatically for both observers. We also examined orientation disparity in UD by presenting a vertical grating to one eye and a horizontal grating to the other and requiring the observer to report which eye received the vertical grating. Observers R.B. and W.M. were able to identify the eye of origin consistently with 1 -cycle/deg gratings of orthogonal orientations.

\section{Discussion}

Considered together, the data presented here provide, at best, mixed support for explanations of UD

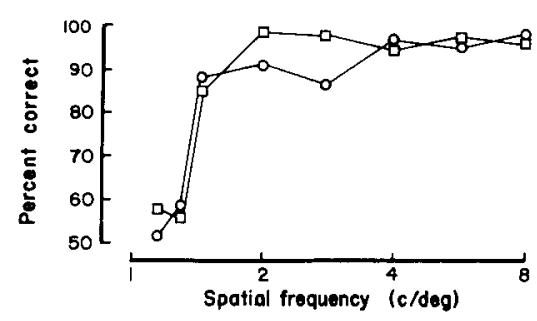

Figure 3. Utrocular discrimination as a function of spatial frequency disparity. Observers R.B. (squares) and W.M. (circles) were required to identify which eye received a 1 -cycle/deg grating when the other eye simultaneously received the spatial frequency shown on the abscissa. $N=100$.

based on oculomotor mechanisms. Considering, first, the negative evidence, stimulus offset proved to be just as robust as stimulus onset, both in terms of successful UD and the accompanying sensation. Moreover, offset performance exhibits the same strong dependence on spatial frequency. Of course, one could argue that grating offset provokes the same, equally potent set of ocular reflexes as grating onset. We find this argument uncompelling, however, in view of the rather marked differences in the engagement of the two eyes at the time of testing with onset vs. offset. We are intrigued by the steady decline in UD performance with decreasing SOA. This indicates that grating offset loses its uniqueness for UD when followed closely in time by an offset in the other eye. It may be possible to capitalize on this temporal resolution limit in future studies of UD.

Equally problematic for an oculomotor hypothesis of UD are the results from the experiment involving binocular stimulation with disparate spatial frequencies and orientations. It is difficult to imagine how spatial frequencies differing by as little as $.15 \mathrm{log}$ units would generate distinctive motor commands. Yet, observers easily judged which eye received the lower spatial frequency with differences this small. These results do indicate that the mechanism responsible for UD is selective for spatial frequency and that its selectivity closely matches the range of spatial frequency disparities that are fusable binocularly. This similarity between UD and fusion is probably not coincidental and is further supported by the finding that observers can distinguish which eye receives a 1-cycle/deg vertical grating when the other eye simultaneously receives a horizontal one. Under steady viewing, these orthogonal gratings generate binocular rivalry.

Of all our results, those from the eye movement experiment are most suggestive of oculomotor involvement. UD performance was impaired, but not abolished, when the observer was required to track the movement of a spot of light across the CRT screen; the extent of impairment was related to dot velocity. Of course, this mode of viewing also introduces tem- 
poral modulation of contrast as the eye sweeps across the stationary grating. If we assume accurate pursuit of the dot by the eye, the temporal modulation for a 1-cycle/deg pattern would range from 2.33 to $11.5 \mathrm{~Hz}$, depending on dot velocity. Other work (Blake \& Cormack, 1979) has shown that temporal frequencies in this range have no influence on UD with steady fixation, so it seems unlikely that the drop in performance during pursuit movements stems from temporal modulation of the pattern on the retina. It is conceivable that the additional demands on attention imposed by this task might have contributed to the impairment in UD, although it is not obvious why such attentional demands would be velocity dependent. It is also possible that some form of saccadic suppression occasioned by tracking served to impair full visibility of the test grating, which in turn might interfere with UD. In fact, observers did note some reduction in the clarity of the grating during pursuit tracking, especially at higher velocities. However, there is no reason to expect this alone to impair performance, since reductions in contrast of a fixated grating have no influence on UD (Blake \& Cormack, 1979).

Thus, it is not unreasonable to attribute the drop in UD performance in the tracking experiment to the engagement of the pursuit eye-movement system, rather than to some concomitant of tracking. Now, presumably, this pursuit task activates oculomotor command centers and extraocular musculature, which in turn would weaken their potential contributions to UD. Based on this presumption, it is a little surprising that forced convergence, which should have produced comparable motor demands, failed to influence UD, unless there is something critical about tracking eye movement. Also puzzling is the relationship between dot velocity and UD. There is no obvious reason why the degree of involvement of oculomotor processes should depend on tracking dynamics. Finally, it is noteworthy that, even in the face of pursuit eye-movements, UD never fell to chance level, as it does reliably in other situations (Blake \& Cormack, 1979). So, at best, the cue for successful UD is only weakened during eye movements. On the balance, the combined results of all these experiments weigh against the hypothesis that oculomotor events are solely responsible for UD. This, in turn, sets the stage for the masking experiments described in the next section.

\section{SPATIO'TEMPORAL PROPERTIES OF UTROCULAR DISCRIMINATION MASKING}

Having unsuccessfully attempted to mask any efferent motor cues, we next attempted to mask afferent cues arising from differential stimulation of the two eyes. We reasoned that successful UD must somehow be based on an afferent neural signature that uniquely specifies left- vs. right-eye stimulation. Such a signature is, at least in principle, an inherent property of an ensemble of visual neurons that differ in terms of ocular dominance. Clearly, neurons equally responsive to stimulation of each eye could not furnish distinctive information about the source of monocular stimulation. On the other hand, neurons that respond differentially with respect to the eyes (the conventional physiological definition of ocular dominance) could, as an ensemble property, signal which of the two eyes received stimulation. In effect, we are conceptualizing the basis of UD as a temporary skewing of neural activity within an ensemble of neurons with varying degrees of ocular dominance. With this in mind, imagine that the activity within this ensemble is uniformly elevated by equal stimulation of both eyes. Such an elevation in overall activity may serve to reduce the degree of skewness produced by monocular stimulation and thereby impair UD. In this section, we demonstrate that a binocular masking target does, indeed, interfere with UD, and we also measure the extent to which this masking effect depends on the spatial and temporal overlap of test and mask.

\section{Masking Display}

For these masking experiments, we used onedimensional noise that could be displayed on the CRTs by feeding the output from a commercial noise generator (Grason-Stadler Model 1285; 20-20-kHz bandwidth with less than $.5 \%$ total harmonic distortion) into the z-axis amplifier. Selected frequencies could be removed from this noise using an electronic filter (Ithaco Model 4302; attenuation factor of $48 \mathrm{~dB}$ / octave from the 3-dB point). This filtered noise could be electronically mixed with the sinusoid used to produce the test target. The combined energy of the mask and test target never exceeded the linear range of the 2-axis amplifier. In some experiments, the mask and test were presented in succession, with stimulus onset asynchrony (SOA) controlled precisely to within an accuracy of $1 \mathrm{msec}$.

\section{Spatial Selectivity}

There are several reasons for believing that the mechanism underlying UD may be selective for spatial frequency, at least to some degree. For one thing, the range of spatial frequencies over which observers can perform successfully on UD is narrower than the range of frequencies that are resolvable (Blake \& Cormack, 1979): whereas the limit of spatial resolution is $40-50$ cycles/deg, the limit for UD is only about $4 \mathrm{cycles} / \mathrm{deg}$, so there is more than a 3-octave range of resolvable frequencies that fall outside the limits of the UD mechanism. Moreover, within this discriminable range, there must be further selectivity 
of some sort, for, as shown in Section 1, observers can correctly identify which eye receives the lower of two spatial frequencies when both eyes receive grating patterns. In order for observers to succeed at this judgment, the two spatial frequencies must differ by at least $15 \%$; for differences smaller than this, UD falls to chance levels.

The purpose of this first series of experiments was to examine in more detail the possibility that UD is mediated by a spatially selective mechanism. In all these experiments, the observer's task was to judge which one of the eyes received a sinusoidal grating. This test grating appeared embedded in a binocularly viewed masking display consisting of one-dimensional random noise. The noise was present continuously, and it resembled bars of a variety of sizes, all jittering across the CRT screen. By filtering the noise, it was possible to vary the spectral overlap between the target and the mask. Presumably, as the range of spatial frequencies contained in the noise overlaps less and less with the range of frequencies that activate the mechanism underlying UD, the debilitating effects of noise on UD performance will be reduced. Noise falling wholly outside the mechanism's bandwidth should produce no impairment in UD, relative to nonmasked performance.

Broadband noise. In a preliminary experiment, we measured UD performance at three spatial frequencies $(1,2$, and 4 cycles/deg) in the presence of broadband noise whose spatial frequency spectrum covered the entire range of spatial frequencies to which the observer was sensitive. The broadband noise was present continuously, and the test grating was exposed for $400 \mathrm{msec}$, including a rise and fall time of $100 \mathrm{msec}$. For each observer, the noise contrast was fixed to produce a criterion level of UD performance. For W.M., the noise energy used was set at an rms voltage equal to that of a sinusoidal grating of $20 \%$ contrast; for R.B., the noise contrast was $10 \%$. The contrast of the test grating was varied over a 1-logunit range, starting at a level at which the test grating was barely visible when superimposed in noise. For purposes of comparison, UD performance was also measured over this same range of test contrasts under nonmasking conditions, in which both eyes viewed an uncontoured raster of luminance equal to the average luminance of the masking noise.

As shown in Figure 4, in the absence of the binocular masking noise the reduction of test grating contrast had no effect on the observer's ability to perform UD. However, when the test pattern appeared embedded in binocular noise, UD performance fell to chance at contrast levels yielding nearperfect performance when there is no masking noise. This drop in UD with masking noise occurs despite the fact that the apparent contrast of the test grating was only modestly affected by the presence of the

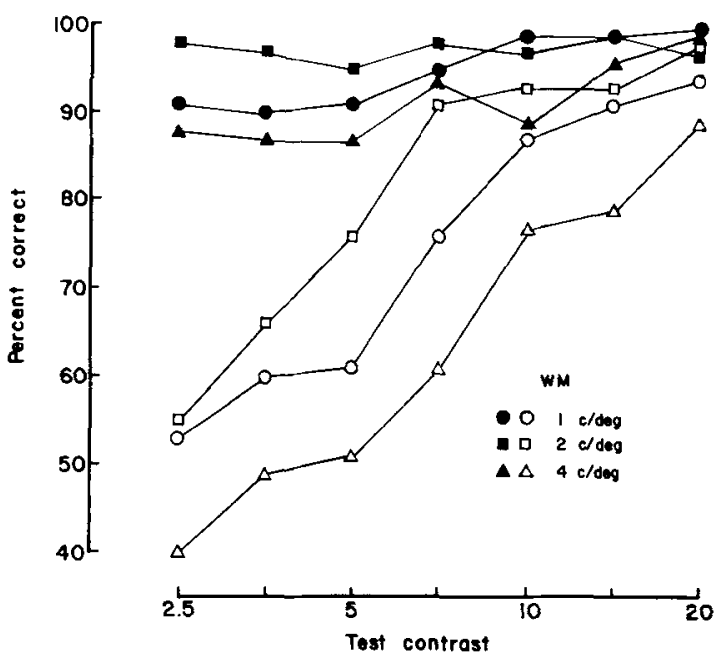

Figure 4. Utrocular discrimination as a function of test contrast for one observer at three spatial frequencies. Open symbols plot performance in binocular visual noise, while filled symbols plot performance in the absence of masking noise. The test pattern was gradually shaped on for 100 msec, held at the nominal contrast for $200 \mathrm{msec}$, and then shaped off for $100 \mathrm{msec}$ in order to avoid the contribution of abrupt transients to UD performance. Each data point is based on 100 trials. Values above $60 \%$ represent significant deviations above chance performance, defined here as 20 .

masking noise, as indicated by subjective reports and confirmed by contrast matching measurements. The same pattern of results was obtained for Observer R.B., with the exception that his nonmasked UD performance at 4 cycles/deg was near chance. Thus, for that observer, no decrease in UD with masking could be measured at this spatial frequency.

It should be stressed that UD performance was impaired by the binocular masking noise even though the low-contrast grating remained clearly visible. It had previously been found that, at low spatial frequencies, excellent UD was always possible whenever the grating could be seen. Reducing the values of energic variables such as the contrast and duration of the test pattern had no effect on UD performance as long as the grating was above the threshold for detection (Blake \& Cormack, 1979). Under conditions of binocular masking, however, the test grating remained visible, but the cue ${ }^{1}$ that normally supports successful UD was absent. This dissociation of UD and grating visibility implies that grating detection and UD are not mediated by identical neural events.

Band-reject noise. Having established that UD could be impaired by binocular masking noise, we next examined the spatial selectivity of this masking effect. The goal of this experiment was to determine just how similar the frequency components in the masking noise had to be to the spatial frequency of the test grating in order to interfere with UD. To ac- 
complish this, we selectively filtered portions of the spatial frequency spectrum from the masking noise in bands centered on the test frequency. This bandreject filtering of the noise produced notches in the noise whose upper and lower cutoff frequencies could be precisely controlled. In all other respects, this experiment was identical to the previous one.

The band-reject masking paradigm is illustrated in the inset of Figure 5. The width of the notch produced in the noise was varied from 0 to \pm 2 octaves. The masking functions displayed in Figure 5 were generated by increasing the notch width in .33-octave steps, always adjusting the total noise power to a constant level. The test grating contrast was maintained at $3.54 \%$, the highest value that gave chance UD performance with broadband masking noise at $20 \%$ contrast. This value was chosen so that any reduction in masking that might occur with filtering would be reflected in an elevation of UD performance to above-chance levels.

Figure 5 shows that the masking of UD is, indeed, selective for spatial frequency. As the notch width in the noise was increased, UD performance improved steadily even though the total noise energy remained constant. In fact, UD performance returned to the unmasked levels when the cutoff of the noise was situated one octave on either side of the test frequency (i.e., a notch width of \pm 2 octaves relative to the test frequency). The results for Observer R.B. were qualitatively similar, except at 4 cycles/deg, at which point his UD performance never climbed much above chance levels even under nonmasking conditions.

This particular strategy for estimating the spatial selectivity of the UD mechanism assumes that the effects of masking noise are symmetrically distributed on either side of the test frequency. The correct-

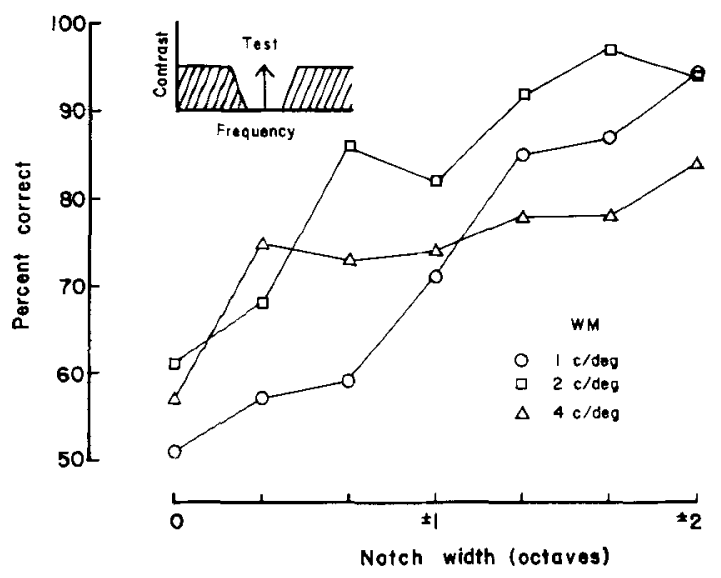

Figure 5. Utrocular discrimination as a function of the width of a rejected band of spatial frequencies in a binocular masking noise. Stimulus conditions were comparable to those in the broadband masking conditions. The inset illustrates the band-reject masking paradigm. ness of this assumption, however, cannot be evaluated using the band-reject paradigm, because the width of the notch of rejected noise was varied simultaneously on both sides of the test frequency. In view of the falloff in contrast sensitivity at low and high spatial frequencies, it is conceivable that these assumptions are violated when testing in these regions of the spatial frequency spectrum. To avoid this possibility and to evaluate the symmetry assumption, masking was measured using two additional varieties of filtered noise, high-pass and low-pass noise.

High-pass and low-pass noise. We were interested in whether, under the binocular masking conditions known to interfere with UD, there would be any asymmetry in the spatial selectivity of this masking effect. The next two experiments were performed to determine whether binocular noise containing only frequencies below the test frequency masked UD more effectively than noise containing only frequencies above the test frequency. The forms of filtering used (illustrated in the insets of Figures $6 a$ and $6 b$, respectively) differ from band-reject filtering in that the noise only contains frequencies above a given cutoff frequency (high-pass noise) or below a given cutoff frequency (low-pass noise). For both types of noise, the roll-off in power spectral density from the nominal cutoff frequency was $48 \mathrm{~dB} /$ octave.

Figure 6a shows that noise situated one octave or more above the test frequency does not impair UD performance. Only when the cutoff of this high-pass noise falls at or below the test frequency was there substantial masking of UD. As the cutoff is further lowered below the test frequency, UD masking increases at the two higher test frequencies. Figure 6b shows the outcome with low-pass noise. Again, it was only when the noise contained frequencies near the test frequency that any appreciable masking of UD occurred. A comparison of the high-pass and lowpass masking functions does not reveal any marked assymetry in the selectivity of UD masking at the two lower spatial frequencies. At 4 cycles/deg, however, there is evidence for asymmetrical masking. In particular, the masking with low-pass noise is more extensive than it is with high-pass noise. ${ }^{2}$ In other words, it seems that UD is not impaired by spatial frequency components that fall outside the range of frequencies in which UD is possible. At the two lower spatial frequencies, the high-pass noise still contains frequencies that are passed by the spatial filter of the UD mechanism.

In general, the results from this first set of experiments indicate that UD is mediated by neural mechanisms that possess a moderate degree of spatial selectivity.

\section{Temporal Properties}

The previous results demonstrate that binocular masking noise can impair UD performance even 


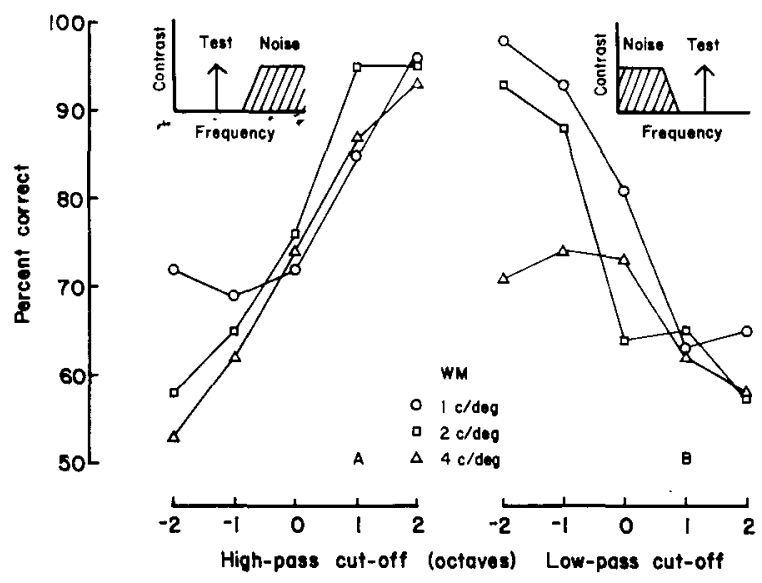

Figure 6. Utrocular discrimination as a function of high-pass and low-pass frequency cutoffs in binocular masking noise for one observer at three spatial frequencies. The insets illustrate the respective masking paradigms.

though the test grating itself is quite visible. According to our intuition concerning UD, this impairment results from the overall increase in activity produced by binocular stimulation within a neural ocular dominance ensemble. In effect, the elevated level of activity produced by a mask swamps the skew of activity within this ensemble produced by the monocular test stimulus, thus diluting the normally salient cue for UD. In the set of experiments described below, we sought to determine just how closely in time the binocular mask must be presented relative to the test grating in order to impair UD. Presumably, there exists some temporal separation beyond which the effects of the mask will have decayed (i.e., the case of mask preceding test) or will have arrived too late to interfere with the test (i.e., test precedes mask). Based on other masking studies (Breitmeyer \& Ganz, 1976) and reaction-time work (e.g., Breitmeyer, 1975), it appears that processing time varies considerably for different spatial frequencies. In view of these findings, we were particularly interested in determining the form of the UD masking function for test gratings of various spatial frequencies.

Asynchronous stimulation and UD. In this experiment, the broadband, binocular noise and the monocular test grating were each pulsed on for $20 \mathrm{msec}$, with abrupt onset and offset $(.1 \mathrm{msec})$. The mask and test were presented at various stimulus onset asynchronies (SOA) ranging from -120 msec (mask precedes test) to +120 msec (test precedes mask). This range of SOA was sampled in 20-msec steps, thus yielding 13 values of SOA. Four different spatial frequencies were tested-.5, 1,2 , and 4 cycles/degalways using broadband noise. Grating contrast was always $10 \%$, and noise contrast was $20 \%$. All trials at a single SOA were completed at each of the four test frequencies before moving to a different SOA value, with the order of the test frequencies randomized at each SOA.

Figure 7 shows that the mask and the test must occur closely in time in order to interfere with UD when the test pattern is of low spatial frequency. When binocular masking noise precedes the test by $80 \mathrm{msec}$ or more, UD is unimpaired at .5 or 1 cycle/deg. At shorter negative SOAs, however, UD has fallen to near-chance levels at these low spatial frequencies, while at 2 and 4 cycles/deg UD remains virtually unchanged. Furthermore, when the mask immediately follows a low-frequency test grating, there is considerably less masking than when the mask immediately precedes the test. This asymmetry is well illustrated at .5 cycles/deg: When the mask follows the test by only $20 \mathrm{msec}$, UD performance rises well above chance, but, when the mask precedes the test, above-chance performance is not evidenced even for SOAs as large as $60 \mathrm{msec}$. The pattern of results is markedly different when the test pattern is higher in spatial frequency. First, UD is well below nonmasked levels even when the noise occurs $120 \mathrm{msec}$ before or after the test; in other words, masking occurs over a much larger range of SOAs for high spatial frequency test targets. This temporally extensive masking effect at higher frequencies may have to do with the fact that at these higher spatial frequencies the UD cue is substantially weaker to begin with under nonmasking conditions. Apparently, this weaker cue is more liable to interference over time.

During the course of this experiment, both subjects reported a rather surprising observation. Under certain conditions, they were able to judge reliably which eye had received monocular stimulation even though

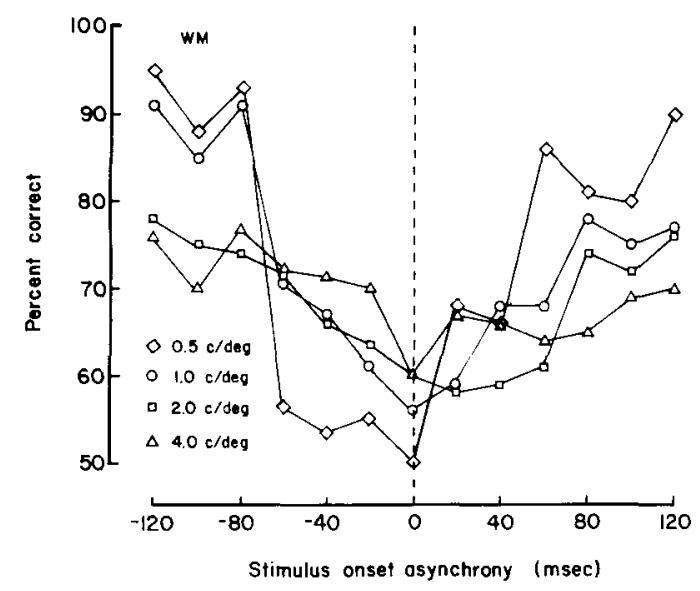

Figure 7. Utrocular discrimination as a function of stimulus onset asynchrony (SOA) for one observer at four spatial frequencies. Negative SOAs Imply that the mask precedes the test target. 
the grating itself was phenomenally invisible, due to masking. This observation seemed potentially significant, for it suggested that those neural events that furnish eye signature information may differ from those involved in actually recognizing a pattern. In order to explore this observation more carefully, the following experiment was performed.

Recognition vs. UD. In this experiment, the 20 -msec pulse of broadband binocular noise of $20 \%$ contrast always preceded the monocular test grating by 120 , 100,80 , or $60 \mathrm{msec}$; these negative SOA values were selected based on the phenomenal reports from the previous experiment. The spatial frequency of the test grating was either .5 or $1 \mathrm{cycle} / \mathrm{deg}$, with the order of frequencies varied randomly from trial to trial. Grating contrast and duration were the same as before, $10 \%$ and $20 \mathrm{msec}$, respectively. Following each trial, the observer was required to report both which eye received the grating and whether the spatial frequency of that grating was "high" ( $1.0 \mathrm{cycle} / \mathrm{deg})$ or "low" ( .5 cycle/deg).

In the absence of masking noise, this spatial frequency identification was trivially simple; over a series of 100 control trials involving no noise, both observers performed without error. When the test grating was preceded by masking noise, however, this part of the dual task was difficult at longer SOAs and impossible at shorter values (the filled symbols in Figure 8 give the results for the spatial frequency identification portion of the task). Yet, despite this inability to identify the grating, both observers could still perform reliably above chance on the utrocular portion of the task, as evidenced by the open symbols in Figure 8. To see if dividing attention between these two tasks contributed to this pattern of results,

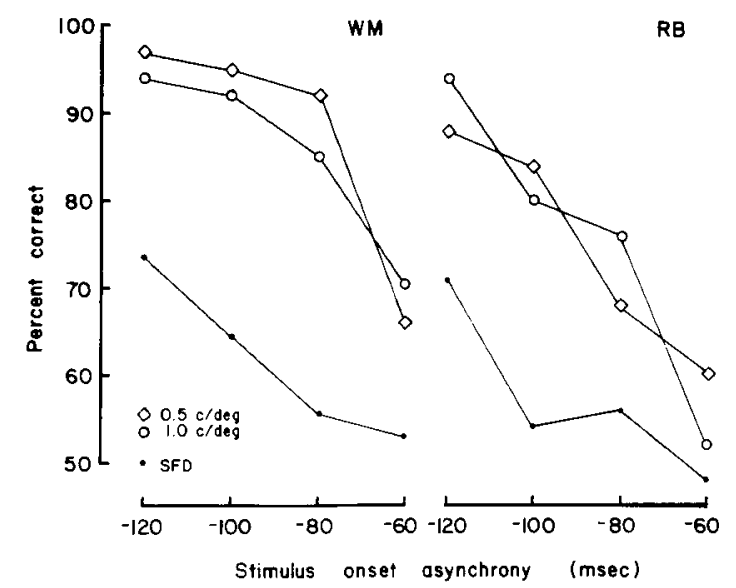

Figure 8. Open symbols refer to utrocular discrimination as a function of negative stimulus onset asynchrony for two observers at two spatial frequencies. Closed symbols refer to spatial frequency identification performance using the same two spatial frequencies as in the utrocular task. we also ran trials in which the observer judged only spatial frequency or judged only the eye stimulated. Results from these trials match very closely the data shown in Figure 8, thus demonstrating that this pattern of results is not peculiar to the dual demands of the task. We analyzed the results from this experiment according to the conditional probabilities of a hit or miss on UD and found no relationship between UD performance and spatial frequency recognition; performance on one task was uncorrelated with performance on the other. These results confirm the subjective impressions from the previous experiment, that the cue mediating UD can exist even when information about the spatial character of the test pattern is insufficient for identifying the spatial frequency of that pattern.

Masking by transients. Our UD/recognition experiment clearly demonstrates that pattern information is not necessary for successful UD, in that UD is possible when spatial frequency recognition is not. This suggests that the cue for UD is furnished by some aspect of grating stimulation other than spatial frequency. Could it be that transients accompanying the brief presentation of a test grating provide a sufficiently robust cue for UD? This idea was advanced by Blake and Cormack (1979), based on the fact that temporal modulation (i.e., flicker) served to enhance performance on a simple UD task. In the following experiment, we attempted to isolate this potential transient cue and to measure its contribution to UD. In particular, we wished to find a binocular masking stimulus that under conditions of steady, or sustained, presentation had minimal effect on UD. We then would present that mask in a pulsed mode that should accentuate transients in the mask. To the extent that transients alone can mediate UD, this otherwise impotent mask should now impair UD by virtue of its pulsed presentation.

For this purpose, dynamic random noise is inappropriate because of the transients always present in this stimulus. Instead, we opted to use a grating pattern as a binocular mask, since it is possible to manipulate the temporal onset and offset of this signal in a well-defined manner.

In this experiment, a masking grating was presented binocularly in either a pulsed or a sustained mode. In the sustained mode, the binocular masking pattern was shaped on gradually over $200 \mathrm{msec}$ and remained at $10 \%$ contrast for $800 \mathrm{msec}$ before the monocular test pattern was presented; this sort of gradual temporal shaping of the mask was designed to eliminate abrupt transients in the onset of the binocular masking pattern. The test pattern was abruptly presented for $20 \mathrm{msec}$ at $7.07 \%$ contrast. Following the onset of the test pattern, the binocular masking pattern remained on for an additional $800 \mathrm{msec}$, after which its contrast was gradually shaped off over 
a 200-msec period, again in the interest of minimizing transients. Thus, a single trial in the sustained condition lasted $2 \mathrm{sec}$. In the pulsed condition, the binocular masking pattern and the monocular test pattern were simultaneously pulsed for $20 \mathrm{msec}$. The aim here was to maximize the transients in the mask. In both conditions, sustained and pulsed, the spatial frequency of the binocular mask was varied over a 2-octave range centered at the test frequency, which was always 1 cycle/deg.

Results from this experiment are summarized in Figure 9, with the open symbols showing UD performance under the pulsed condition and the filled symbols showing the results for the sustained condition. In the sustained condition, masking gratings removed by 2 octaves above or below the test frequency did not impair UD, but UD performance dropped as the spatial frequency of the binocular masking pattern approached that of the test pattern. This outcome merely confirms the earlier conclusion that UD masking in a sustained mask is spatially selective. However, in the pulsed condition, an entirely different masking function was obtained. At mask frequencies below the test, there was a substantial increase in the extent of the UD masking relative to that obtained under the sustained condition. At mask frequencies above the test, there was no systematic change in the extent of masking relative to the sustained condition. These results demonstrate that the introduction of transients into an otherwise ineffective binocular masking pattern (i.e., one that does not provide a spatial masking effect) can produce a potent masking effect on UD, depending on the spatial frequency of that masking stimulus.

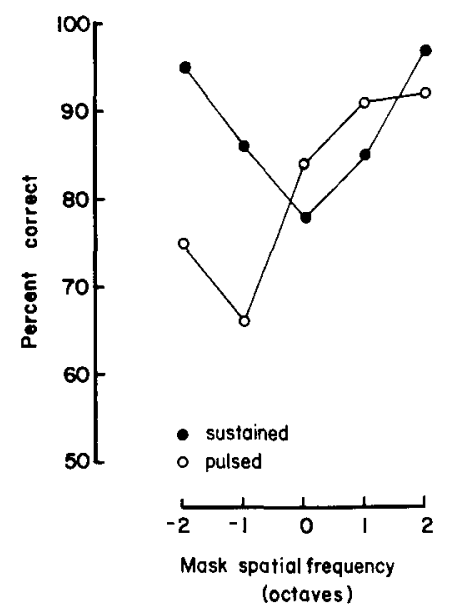

Figure 9. Utrocular discrimination performance under sustained and pulsed presentation of a binocular masking grating whose spatial frequency was varied over a 2-octave range centered at the 1-cycle/deg test frequency.

\section{GENERAL DISCUSSION}

It has previously been proposed (Blake \& Cormack, 1979) that eye signature information might be contained in the overall pattern of activity among cortical neurons with varying degrees of ocular dominance. In effect, the cue for successful UD may involve a skewness in activity within this neural ensemble. The present results have led us to refine this conceptualization of the mechanism underlying UD. For one thing, we need to incorporate into this mechanism a degree of spatial selectivity, as indicated by the simultaneous masking experiments. Yet, at the same time, it appears that this spatially selective mechanism may not necessarily be involved in pattern identification. Rather, there is reason to think that this mechanism mediates successful UD via contrast transients. In the following discussion, we shall consider each of these points in turn.

Our revised conceptualization of the UD mechanism can be described as follows. Neural activity is generated within a population of neurons that vary in terms of their preferred spatial frequency and their ocular dominance. Monocular stimulation produces a skewing of the activity across the ocular dominance dimension, reaching its maximum at the test spatial frequency and falling off from this point in either direction in the spatial frequency dimension. It is this skewness that provides the cue for UD. Now, suppose both eyes view a binocular masking pattern. That mask should result in an overall increase in activity across the entire range of ocular dominance; the spread of activity over the spatial frequency dimension would depend on the band of spatial frequencies contained in the noise. According to this hypothesis, UD is impaired by the masking stimulus when its resulting activity obscures the incremental activity due to the presentation of a monocular test pattern. The extent of its interference with UD is related to the degree of overlap between the envelope of neural activity due to the masking and that due to the test. The UD mechanism is seen as a detector of skewness across ocular dominance. It serves to detect skewness relative to the overall envelope of neural activity resulting from background binocular activity.

Despite the spatial selectivity of the UD mechanism, there is reason to believe that the neural events underlying UD are not identical to those mediating grating recognition. For one thing, we know there are many patterns (i.e., those composed only of high spatial frequencies) that are perfectly detectable and distinguishable from one another and yet that cannot support successful UD. This dissociation of detection and UD was further highlighted in the simultaneous masking experiment. Here it was found that binocular noise could abolish successful UD even though 
the test grating itself was still visible. So, simply being able to see a stimulus fails to guarantee the ability to judge with which eye one is seeing it. Even more to the point, we also found that there are conditions in which it is impossible to identify the spatial frequency of a grating, while at the same time it is possible to judge reliably which eye received the grating. In view of this dissociation of pattern visibility and UD, we are led to consider another aspect of grating stimulation as the requisite cue-namely, the transient that accompanies grating onset.

There are several reasons why we find this hypothesis appealing. First, other types of psychophysical evidence indicate that the transient response to grating patterns is considerably more pronounced at low spatial frequencies (e.g., Lupp, Hauske, \& Wolf, 1978). This, of course, is also the region of the spatial frequency spectrum in which the UD cue is salient, and the task is, therefore, simple. A second reason for believing that transients may be crucial for UD is provided by the outcome of our simultaneous masking experiment with pulsed vs. sustained masks. Recall that UD was more effectively masked when the masking stimulus was presented in a manner that exaggerated its temporal onset and offset; a weak mask could be bolstered in strength by pulsing it rather than gradually introducing and withdrawing it. This suggests that the transient component of the pulsed mask was interfering with the normally salient cue for UD.

Assuming that contrast transients do generate the cue for UD may also shed some light on the basis of the asymmetrical masking functions seen in Figures 7 and 9. Recall that the introduction of transients into a binocular grating mask impaired UD when that mask was as much as two octaves below the test frequency, but had no effect when the mask was two octaves above the test. Also recall from the successive masking study that there were notable differences in the range of SOAs over which masking occurred for different test frequencies. As we shall argue, these kinds of asymmetries may reflect the differing amounts of time necessary for processing different spatial frequencies. This argument is developed in the following paragraph.

To begin, it is well established that reaction time (RT) to the onset of a sinusoidal grating increases with spatial frequency (Breitmeyer, 1975). While it is arguable whether this variation in RT reflects conduction latency differences in sustained vs. transient pathways (Lennie, 1980), no one questions that, for gratings of equivalent physical contrast, lower spatial frequencies are processed more quickly than are higher ones. Now, with this fact in mind, consider the results from the last experiment, which utilized pulsed binocular gratings as a mask. Why did the mask of lower spatial frequency impair UD, while the one of higher spatial frequency did not? With a low-frequency mask, there is sufficient time for the binocular response to its transients to reach the UD mechanism before the monocular response to the more slowly processed test frequency. However, when the binocular masking grating is two octaves above the test frequency, the monocular onset transient produced by the test grating reaches the UD mechanism first, far enough in advance of those in the masking pattern to preclude a masking effect altogether. This line of reasoning also provides a qualitative account of the results from our successive masking experiments.

Recall that Figure 7 illustrates the effects of a 20-msec pulse of broadband masking noise on UD performance at various SOAs. (Keep in mind that negative SOAs indicate that the onset of the masking noise precedes the onset of the 20 -msec test pattern.) UD performance falls to chance levels for the different test spatial frequencies at different SOA values. In particular, at .5 cycles/deg, UD falls to chance at $-60 \mathrm{msec}$ SOA, 1-cycle/deg performance reaches chance at $-20 \mathrm{msec} \mathrm{SOA}$, and, at 2 and 4 cycles $/ \mathrm{deg}$, UD falls to chance only when the masking noise is presented simultaneously with the test. In other words, the temporal extent of forward masking varies directly with the spatial frequency of the test. Lower spatial frequencies, by virtue of their faster processing time, can occur later in time following the mask and still suffer interference from the mask. This analysis makes the quite reasonable assumption that the response to the onset of the masking noise is constant for all SOA conditions.

The same kind of conclusion emerges upon examination of the data at positive SOAs (i.e., backward masking). In this case, the analysis focuses on the minimum amount of time by which the test must precede the mask in order to yield above-chance performance on UD. At .5 cycles/deg, successful UD is found for all positive SOAs, indicating that this low spatial frequency is arriving at the UD mechanism sufficiently rapidly to beat the swamping effect of the binocular mask. At $1 \mathrm{cycle} / \mathrm{deg}$, the test must precede the mask by at least $40 \mathrm{msec}$ to avoid complete masking, and, at 2 cycles/deg, this minimum necessary lead time grows to $60 \mathrm{msec}$. The results at 4 cycles/ deg are difficult to interpret, owing to the fact that UD performance never really climbs much above chance for this observer and was always at chance levels for the other observer.

So, in summary, the temporal properties of UD masking, the effectiveness of pulsed masks, and the low-pass spatial character of UD all point to the involvement of transient responses as a critical cue in UD. More specifically, it appears that the transient component of the grating onset response passes through the low-pass spatial filter of the transient mechanism. 
The neurons that constitute the ocular dominance system responsible for UD receive the output of this filter as well as the sustained component of the response to the test pattern. Thus, only those neurons sensitive to spatial frequencies below the cutoff of the transient mechanism receive strong transient stimulation. Therefore, even though neural activity may be skewed across ocular dominance by the monocular presentation of a grating above 4 cycles/deg, the skew has a negligible transient component, and thus the UD cue cannot be generated. ${ }^{3}$

\section{REFERENCES}

BlAKe, R., \& CoRmack, R. H. On utrocular discrimination. Perception \& Psychophysics, 1979, 26, 53-68.

Breitmeyen, B. G. Simple reaction time as a measure of the temporal response properties of transient and sustained channels. Vision Research, 1975, 15, 1411-1412.

Breitmeyer, B. G., \& Ganz, L. Implications of sustained and transient channels for theories of visual pattern masking, saccadic suppression, and information processing. Psychological Review, 1976, 83, 1-36.

Enoch, J., Goldmann, H., \& Sunga, R. The ability to distinguish which eye was stimulated by light. Investigative Ophthalmology, 1969, 8, 317-331.

Georgeson, M. A., \& Sullivan, G. D. Contrast constancy: Deblurring in human vision by spatial frequency channels. Journal of Physiology (London), 1975, 252, 627-656.

Hubel, D. H., \& Wiesel, T. N. Receptive fields and functional architecture of monkey striate cortex. Journal of Physiology (London), 1968, 195, 215-243.

Lennie, P. Perceptual signs of parallel pathways. Philosophical Transactions of the Royal Society of London, B, 1980, 290, 23-37.
Lupp, U., Hauske, G., \& Wolf, W. Different systems for the visual detection of high and low spatial frequencies. Photographic Science and Engineering, 1978, 22, 80-84.

SMITH, S. Utrocular or "which eye" discrimination. Journal of Experimental Psychology, 1945, 35, 1-14.

Templeton, W. B., \& Green, F. A. Chance results in utrocular discrimination. Quarterly Journal of Experimental Psychology, $1968,20,200-203$.

\section{NOTES}

1. As described in Section 1, this cue for UD can be characterized as a "feeling in the eye." In the present masking experiments, this has continued to be the cue upon which UD was based. Attempts to learn other strategies for successful UD have met with no success.

2. With high-pass noise above 4 cycles/deg, the mask energy falls within a region of the spatial frequency spectrum in which observers are relatively less sensitive. Could this fact alone be responsible for the reduction in masking for this condition? We believe this is unlikely, because the contrast of the noise was still considerably above threshold; indeed, the apparent contrast of this high-pass noise was quite similar to that of low-pass noise, suggesting a sort of contrast constancy of the type documented by Georgeson and Sullivan (1975).

3. An alternative explanation of the spatial frequency effect on UD might be developed in terms of changes in the ocular dominance distribution at higher spatial frequencies. However, to our knowledge there is no evidence suggesting any systematic variation in the extent of binocular interaction with spatial frequency. Since such phenomena as interocular transfer of visual aftereffects and binocular summation do not show any appreciable change with increasing target spatial frequency, we are discouraged from developing such an alternative explanation for the falloff in UD performance at high spatial frequencies.

(Manuscript received January 19, 1981; revision accepted for publication August 10, 1981.) 